\title{
Arrinconados por la realidad: Menores de circuito
}

\section{Cornered by reality: Circuit minors}

José A. Moreno Mena* y Rosa María Avedaño Millán**

\section{Resumen}

Este trabajo analiza un segmento de la migración infantil al que se le ha llamado "menores de circuito". Se trata de niñas, niños y adolescentes migrantes no acompañados que cruzan la frontera por diversos motivos y se les relaciona con bandas delictivas, de trata, traficantes de humanos y drogas. El propósito es hacer visible el problema y reflexionar sobre los riesgos y el estado de vulnerabilidad social que vive esta población. Mediante revisión bibliográfica, hemerográfica y de estadísticas oficiales, así como entrevistas a informantes clave se identifica y caracteriza a esta categoría de migrantes. Se concluye que es necesaria la revisión de los marcos actuales de protección a los niños migrantes y la creación de un protocolo de actuación de las autoridades que tienen contacto con ellos a fin de velar por el interés supremo del menor.

Palabras clave: niñas, niños y adolescentes migrantes, frontera norte, derechos humanos, vulnerabilidad, riesgo.

Recibido: 26 de mayo de 2014.

Aprobado: 18 de noviembre de 2014.

\begin{abstract}
The present study examines a category of migrant children named "circuit minors." This category includes unaccompanied migrant children and adolescents who cross the border continuously for various reasons and are typically associated with criminal gangs and human and drug traffickers. The aim of this paper is to highlight the problem and consider the risks and the state of social vulnerability in which this population lives. These categories of children and adolescent migrants are identified through a review of the literature, newspaper archives, and official statistics. The study draws on interviews with key informants to characterize this population. We conclude that there is a need to review existing frameworks for the protection of migrant children and to create an action protocol for officials who have contact with these children to ensure their best interests.

Keywords: migrant children, Northern border, human rights, vulnerability, risk.
\end{abstract}

* Universidad Autónoma de Baja California, Instituto de Investigaciones Sociales. Dirección: Blvd. Benito Juárez s/n, Unidad Universitaria, Edificio de Vicerrectoría, $3^{\circ}$ piso, Col. Ex ejido Coahuila, C.P. 21280, Mexicali, Baja California, México. Correo electrónico: jmoreno@uabc.edu.mx

** Universidad Autónoma de Baja California, Facultad de Ciencias Humanas. Correo electrónico: rosyaven@hotmail.com 
José A. Moreno Mena y Rosa María Avedaño Millán / Arrinconados por la realidad: Menores de circuito

\section{Introducción}

En estudios recientes sobre la migración de niñas, niños y adolescentes ${ }^{1}$ (en adelante NNA) mexicanos, con frecuencia se menciona la existencia de un segmento de la niñez migrante al cual el Sistema Nacional para el Desarrollo Integral de la Familia (DIF) ha denominado "menores de circuito" o NNA de circuito (DIF-Sonora, 2013; DIf-Tamaulipas, 2013), mientras que la prensa nacional los menciona como "polleritos" o "enganchadores" (Pérez, 2012).

En ambos casos, se refiere a niños, niñas y adolescentes fronterizos que cruzan de manera recurrente e indocumentada a Estados Unidos con diversas motivaciones. Desde hace aproximadamente una década, algunos analistas habían detectado la existencia de este grupo de NNA y los riesgos que corrían, sin embargo, las instituciones gubernamentales encargadas de la protección infantil, tanto mexicanas como estadunidenses, han optado por ignorar su suerte, a pesar de que estos menores son los que están en situación de mayor riesgo social debido al tipo de actividades en las que se les involucra.

Este trabajo tiene varios objetivos: el primero es visibilizar el fenómeno de la niñez migrante a partir de estudios y datos estadísticos; el segundo, es reflexionar sobre los riesgos y el estado de vulnerabilidad social que tiene esta niñez; y en tercero, hacer algunas recomendaciones que contribuyan al establecimiento de políticas públicas para su atención. Para ello recurrimos al análisis de documentos oficiales e informes de organizaciones civiles, estadísticas de repatriación, entrevistas a informantes claves y notas hemerográficas, así como documentación bibliográfica. Partimos del supuesto de que este segmento de nNA migrantes, es el que tiene mayor vulnerabilidad social al ser desdeñado por las instituciones del Estado mexicano y arrojado al crimen por una realidad social que los arrincona sin proporcionarles oportunidades para desarrollarse de manera integral como seres humanos. Partimos del concepto de vulnerabilidad social "como una condición social de riesgo, de dificultad, que inhabilita e invalida, de manera inmediata o en el futuro, a los grupos afectados, en la satisfacción

${ }^{1}$ Cabrera y Valdéz-Gardea (2013) mencionan el fenómeno indirectamente cuando hacen alusión a los menores que se encargan de trasladar a los migrantes por el desierto de Sonora. 
de su bienestar — en tanto subsistencia y calidad de vida- en contextos sociohistóricos y culturalmente determinados" (Perona, Crucella, Rochi y Robin et al., 2000, párr. 17).

El trabajo lo dividimos en dos grandes apartados: en un primer apartado presentamos el estado de la cuestión de los estudios de NNA ubicando a los menores de circuito en la literatura y las estadísticas; en un segundo apartado la situación de riesgo y violencia de la que son objeto y que prevalece en la frontera norte y la intervención con los menores de circuito. Finalmente, emitimos, a modo de conclusión, algunas propuestas sobre los marcos de protección que debería tener este tipo de NNA.

\section{Los estudios sobre NNA y los/as menores de circuito}

Los estudios sobre la migración de niñas, niños y adolescentes mexicanos hacia los Estados Unidos han sido escasos en los análisis académicos. Ramos Tovar (2009, pp. 37-38) sostiene que las investigaciones sobre el tema, más bien han centrado la mirada en las características laborales y sociodemográficas de los migrantes, sus redes, los lugares de origen y destino, las remesas, y últimamente, en la incorporación de las mujeres.

En el caso que nos ocupa, las niñas, niños y adolescentes no acompañados mexicanos (NNA) a partir de la década de los noventa aparecen en los estudios de la migración. La preocupación por el incremento vertiginoso y la vulnerabilidad de los niños y las niñas comenzó a ser tema de discusión de las instituciones internacionales y esto llegó a interesar a los académicos. Según Méndez (2000), fue De la Rosa quien elaboró uno de los primeros estudios sobre el tema en los años noventa; este autor, a partir de los datos de la Casa del Migrante de Tijuana, da cuenta del proceso migratorio de los menores en la frontera México-Estados Unidos estableciendo una caracterización de los NNA no acompañados atendidos en ese centro.

Posteriormente, la Comisión Nacional de Derechos Humanos (CNDH) realizó una encuesta en varias ciudades fronterizas por donde se efectuaba la repatriación de NNA (1993) con la intención de tener un perfil de la niñez migrante.

En 1998 la Academia Mexicana de Derechos Humanos, Albergue del Desierto y el Centro de Apoyo al Trabajador Migrante (1998), junto con 
otras organizaciones civiles de Baja California, presentaron un diagnóstico sobre los NNA en la ciudad de Mexicali, en el libro titulado La esperanza truncada. En ese entonces, Mexicali era la ciudad fronteriza del norte de México más importante de repatriación de NNA y constituía el referente de la migración de esta población.

En el 2001 López y Villaseñor (2001) escribieron algunos ensayos relacionados con los NNA, donde hacen énfasis sobre los riesgos que corrían los menores en ese entonces, especialmente el fallecimiento.

Los NNA eran invisibles en los datos de los migrantes en general y en algunas ocasiones se les relacionaba con la migración femenina, de tal forma que era imposible tener datos confiables del número de nNA que cruzaban hacia Estados Unidos de América (en adelante EUA) por el norte mexicano o se encontraban albergados en las fronteras a la espera de cruzar. ${ }^{2}$ Los únicos que poseían datos eran los consulados mexicanos en los estados fronterizos, puesto que ellos intervenían directamente en el proceso de repatriación junto con las organizaciones civiles de apoyo a NNA migrantes. Cabe destacar que fueron estas últimas las más preocupadas por sistematizar datos sobre esta población infantil. La difusión de sus cifras sirvió a las autoridades para desarrollar políticas de atención hacia la niñez migrante (Academia Mexicana de Derechos Humanos, Albergue del Desierto y Centro de Apoyo al Trabajador Migrante, 1998).

La falta de datos oficiales era un reclamo de las organizaciones civiles y del mundo académico, por lo que el Instituto Nacional de Migración (INM) comenzó a difundir sus estadísticas de repatriados mexicanos de Estados Unidos en el 2001, ya con la visión de género y edad, donde aparecían desglosadas las cantidades de NNA reportadas por los consulados. En dichas estadísticas hay hasta la fecha algunas omisiones de información, especialmente respecto de los NNA que no han cruzado y se encuentran bajo el resguardo de las organizaciones civiles.

Un referente importante para el entendimiento de los NNA fue el estudio sobre 11 ciudades de la frontera norte de México realizado por Gallo ${ }^{3}$

${ }^{2}$ La invisibilidad de la migración NNA ya ha sido estudiada en otros países; por ejemplo, Torrado (2012) identifica este fenómeno en la migración que ocurre en España procedente de África.

${ }^{3}$ El estudio se hizo en el marco del Proyecto Interinstitucional de Atención a Menores Fronterizos que se inició en 1996 con el convenio entre el Sistema Nacional para el 
(2004) con el propósito de analizar el procedimiento interinstitucional seguido a niñas, niños y adolescentes migrantes que viajaban sin compañía de sus familiares. El análisis incluía la normatividad vigente relacionada con el fenómeno migratorio infantil, lo que dio la pauta para que distintas instituciones gubernamentales comenzaran a retomar el tema. Además sirvió para hacer recomendaciones con la finalidad de contribuir a mejorar la gestión migratoria y para armonizar el marco normativo con la Convención sobre los Derechos del Niño.

Posteriormente, dado el crecimiento de NNA repatriados por Sonora, la investigadora de El Colegio de Sonora, Valdéz-Gardea (2013), convocó al I Encuentro Internacional de Migración y Niñez Migrante en 2007, realizándose hasta la fecha cuatro encuentros que han generado una gran cantidad de materiales e insumos sobre el tema de los NNA; además, los encuentros han permitido abordar diferentes perspectivas de esta población. ${ }^{4}$

Conviene destacar dos estudios recientes por la variedad de los temas que abordan, el primero es el de Ramírez et al. (2009). En ese documento, los autores distinguen distintas rutas migratorias de los NNA y concluyen que la migración de niños y niñas, al igual que la de adultos, sigue trayectos migratorios claramente establecidos, que unen el lugar de origen con las ciudades fronterizas en donde se intentará o se hará el cruce. Uno de los aspectos más interesantes del trabajo de Ramírez et al. (2009), es que proporciona elementos para caracterizar a los menores migrantes, al proponer categorías para clasificarlos, debido a que según su opinión, las utilizadas hasta ese momento resultaban insuficientes.

El segundo estudio fue elaborado por la organización civil Appleseed (2011); resulta una investigación pormenorizada sobre los procedimientos y las prácticas que llevan a cabo las autoridades estadunidenses y mexicanas para la repatriación de los NNA. Centra su observación en los procedimientos que atentan contra el interés supremo del niño y hace una serie de recomendaciones en materia de política pública a los sistemas de protección de la niñez de los dos países.

Desarrollo Integral de la Familia y el Fondo de las Naciones Unidas para la Infancia, al que se sumaron posteriormente la Secretaría de Relaciones Exteriores y el Instituto Nacional de Migración.

${ }^{4} \mathrm{Al}$ respecto, consúltese Valdéz-Gardea (2008 y 2013). 
José A. Moreno Mena y Rosa María Avedaño Millán / Arrinconados por la realidad: Menores de circuito

\section{¿Quiénes son los menores de circuito?}

En diversos estudios sobre la niñez migrante, se utilizan varios términos para referirse a los NNA. En algunas ocasiones se refieren a menores migrantes no acompañados, en otras a niños y niñas migrantes no acompañados, o bien ambos como sinónimos. Según Ramírez et al. (2009), el Programa de Menores no Acompañados en Europa prefiere utilizar el término de "niñas y niños separados", en vez de "no acompañados", tal como lo había establecido anteriormente el Alto Comisionado de las Naciones Unidos para los Refugiados (ACNUR, 1989), para identificar a la niñez que forma parte de los flujos migratorios que cruzan o intentan cruzar la frontera norte, en manos de personas que no son sus padres o cuidadores habituales. Según Ramírez et al. (2009), el argumento fundamental del Programa de Menores no Acompañados en Europa, era que ese concepto define mejor el principal problema que está afrontando esa población infantil; es decir, los NNA se encuentran sin los cuidados y la protección de sus padres y de su tutor legal, y como consecuencia, sufren, a nivel social y psicológico, los efectos de esa separación. Y aunque lleguen "acompañados”, los adultos que les custodian no son necesariamente los apropiados o capaces de asumir la responsabilidad de cuidar a esos menores.

En este trabajo, preferimos utilizar las definiciones que presenta la Convención sobre los Derechos del Niño del Alto Comisionado (1989), que en su artículo 1o define al niño como aquella persona menor de dieciocho años de edad, salvo que en virtud de la ley que le sea aplicable, haya alcanzado antes la mayoría; y el término de niña, niño o adolescente no acompañado migrante (NNA), para referirse a los niños que cruzan la frontera solos, así como los que lo hacen con sus pares o con personas que no son sus familiares y que los abandonan a su suerte en los puntos de cruce donde son aprehendidos por las autoridades de inmigración de Estados Unidos (Hernández, 2012).

En esta misma categoría de niñez migrante entran los niños y niñas que emigran solos a la frontera con la intención de cruzar hacia EUA sea para reunificación familiar, cuestiones laborales o de otra índole. De la misma forma, a los niños y niñas interceptados en territorio estadunidense y que fueron repatriados solos o con sus amigos. Desde esa visión, también 
entrarían los llamados "menores de circuito", que aparecen esporádicamente en algunos estudios académicos.

En 2012, la red de organizaciones civiles Programa de Defensa e Incidencia Binacional (en adelante PDIB) promovió un Amicus Curieae ${ }^{5}$ ante la Corte Interamericana de Derechos Humanos con la siguiente clasificación de NNA en circuitos:

Son aquellos que cruzan de manera recurrente e indocumentada a EUA por motivos que no tienen que ver con la búsqueda de trabajo ni la reunificación familiar. Pueden clasificarse en dos conjuntos: un tipo de NNA que son originarios de las ciudades fronterizas mexicanas, que suelen pasar la frontera como una experiencia de vida y la decisión de cruzar tiene una recompensa en el plano simbólico: la de adquirir experiencia, demostrar madurez y/o valentía. El segundo tipo incluye a niños, niñas y adolescentes menores de edad que se encuentran involucrados con redes de tráfico de personas para que guíen el paso de migrantes por la frontera, o bien, con grupos del crimen organizado para el trasiego de droga (Programa de Defensa e Incidencia Binacional, 2012, p. 11).

El Programa de Defensa e Incidencia Binacional (2012) ha señalado que los menores en circuito cruzan la frontera norte para saber qué tanto pueden lograr al momento del cruce, lo que les resulta satisfactorio aun si son repatriados, ya que la decisión de cruzar tiene una recompensa en el plano simbólico: la de adquirir experiencia, demostrar madurez y/o valentía. Ya en los estudios de López (2003) sobre los migrantes de Michoacán, se menciona el "rito de paso" que muchos niños, niñas y adolescentes llevan a cabo, que significa el "llegar a la edad de ir al norte". De acuerdo con López (2003), en el proceso de socialización, los niños y las

${ }^{5}$ Amicus Curiae es una expresión latina que significa literalmente "amigos de la corte" y su objetivo es abrir la posibilidad a terceros que no son parte de un litigio, pero que poseen un interés demostrable y justificado en la resolución de éste, a promover voluntariamente una presentación que contenga una opinión técnica mediante la cual aporten elementos que puedan resultar jurídicamente trascendentes al momento de que el juzgador resuelva sobre la materia del litigio (Nexos, 2012). La información proporcionada puede consistir en un escrito con una opinión legal, un testimonio no solicitado por parte alguna o un informe en derecho sobre la materia del caso. En el caso citado, se presenta como un testimonio de una organización civil. 
niñas aprenden los elementos de la migración, y acceden a un capital simbólico que les permite participar en experiencias migratorias, de los conocimientos, saberes y estilos de vida que les sirven como marco de referencia para actuar. Aun y cuando nunca han ido a los EUA ya tienen referentes de otros adolescentes que han emigrado y de sus propios padres.

Le Goff (2012) ha registrado que algunos NNA viajan motivados por la aventura y para "conocer el otro lado", como si se tratara de un rito de aprendizaje adolescente; mientras que Vilaboa (2006) indica que 3.2\% de NNA entrevistados en su estudio expresó que su intento de cruzar de manera indocumentada a los Estados Unidos, fue para ir de "paseo". La otra forma que adquieren los menores de circuito, de acuerdo con el Programa de Defensa e Incidencia Binacional (2012), es la de los niños, niñas y adolescentes que se encuentran involucrados - por propia voluntad o en contra de ella- con redes de tráfico de personas para que guíen el paso de migrantes por la frontera, o bien, con grupos del crimen organizado para el trasiego de droga al vecino país del norte. De igual manera, aquellos que son engañados o enganchados para que presten servicios sexuales a adultos.

Sobre las actividades que frecuentemente llevan a cabo o que son obligados a realizar los menores de circuito, se encuentra la de:

[...] ubicar posibles víctimas en los propios albergues dispuestos por las autoridades mexicanas u operados por organizaciones civiles para atender NNA migrantes, como paso previo a ser devueltos a su lugar de origen o en los puntos de cruce establecidos por coyotes y polleros, para ser explotados laboral o sexualmente. Algunos de estos, menores de 18 años, se encargan de enganchar a niños a quienes convencen de escapar del albergue y les ofrecen cruzarlos por la frontera para, en realidad, dejarlos en manos de organizaciones criminales (Pérez, 2012).

De ahí que se señale que el fenómeno de los nNA ha propiciado una nueva figura, no sólo en el entramado de la migración indocumentada sino también al interior del crimen organizado, pues a raíz de su vinculación con esos grupos, se les señala despectivamente como "niños y niñas de circuito", "enganchadores" o "polleritos" (Pérez, 2012), o bien "coyotitos", "guías" o "halcones". 
Como vemos, la existencia de los menores de circuito representa una realidad que no se puede ocultar. En la literatura sobre nNA podemos visibilizar a éstos en la siguiente clasificación propuesta por Ramírez et al. (2009):

Menores que cruzan la frontera para reunirse con su familia: que por lo común, están acompañados por algún familiar, amigo o son entregados a un coyote para el cruce.

Menores de entre 12 y 13 años de familia pobre. Conjunto de menores, que generalmente son los de mayor edad en su familia, motivo por el que se sienten presionados y se apresuran para empezar a contribuir a los gastos familiares.

Menores que dejan a su familia por espíritu de aventura y abandonan la escuela, o menores que tienen problemas en el seno familiar, con los papás, padrastros o tutores.

En esta clasificación aparece en el último párrafo un tipo de NNA que pueden ser equiparados a los menores de circuito.

En otro estudio, Méndez (2000) encontró que la red de Casas YMCA ${ }^{6}$ para Menores Migrantes (СYмm) también hizo un esfuerzo por clasificar a los NNA en distintos rubros, que permitieran ir conociendo las problemáticas que les rodean. De tal forma que identificaron las siguientes tres categorías:

Migrantes transitorios. Son los menores definidos así por sus fuertes lazos familiares y con domicilios en Estados Unidos.

Migrantes nómadas. No cuentan con algún familiar, o bien, en algunos casos no proporcionan la información que permita averiguar el paradero de los mismos y con ello canalizarlos con alguna persona adulta. Por tanto, no se logra reintegrarlos a un ambiente familiar, y son los que presentan una mayor reincidencia para cruzar a Estados Unidos.

Migrantes transfronterizos. Son aquellos menores que viven en ciudades fronterizas mexicanas y no tienen un núcleo familiar definido. Generalmente se han desarrollado en ambientes callejeros, sin el cuidado y protección de familiares. Las razones de su cruce son inciertas y tampoco desean regresar a sus hogares, o con sus familiares (p. 17).

${ }^{6}$ Por sus siglas en inglés: Young Men’s Christian Association. 
José A. Moreno Mena y Rosa María Avedaño Millán / Arrinconados por la realidad: Menores de circuito

En esta clasificación de YMCA también se puede observar la presencia de menores de circuito en la categoría de menores transfronterizos y menores nómadas.

Diversos son los escenarios de los niños migrantes; Mancillas (2009) menciona que hay niños migrantes en tránsito, solos o acompañados y transfronterizos, que tienen movimiento constante en la frontera: ello para adquirir experiencia o por diversión, formando parte de la dinámica cultural. Los define como:

[...] los polleritos, los cuales son menores de edad que ayudan a otros migrantes a pasar la frontera. Los repatriados son aquellos que fueron descubiertos por las autoridades estadounidenses y que son regresados a territorio mexicano. La cuarta condición es la de los menores fronterizos, que son migrantes o repatriados que se han establecido temporal o permanentemente en la frontera. Se pueden dedicar al ambulantaje o ser "niños de la calle". Algunos llegan a situarse en túneles de drenaje en la frontera entre Nogales, Sonora, Nogales y Arizona (2009, p. 231).

En 2006, en el seminario "Niñez migrante no acompañada en la frontera norte: Repatriación, protección y atención”, llevado a cabo en El Colegio de la Frontera Norte en la ciudad de Tijuana, se mencionó el tema al señalarse que:

Se reconoce la existencia de dos tipos de niños y niñas migrantes: aquellos que provienen del sur del país, cuyo interés principal es el cruce hacia los EU, y aquellos que viven en la región y que, en realidad, pueden tener como práctica común el servir como guías en el cruce indocumentado de personas hacia EU (2006, p. 9).

Por su parte, Vilaboa (2006), en una investigación realizada en la ciudad de Nogales, Sonora, encontró que 1.6\% de los NNA entrevistados correspondió a un grupo de residentes fronterizos, quienes en su mayoría se reservaron los motivos para haber tratado de ingresar a Estados Unidos; mientras que otro $1.6 \%$ expresó que su ingreso se debió a que habían traficado con drogas. 


\section{Visibilización de los menores de circuito en las estadísticas}

Las estadísticas oficiales regularmente han omitido el tema de los menores de circuito ya que no registraban las características de nNA, aun y cuando en los años noventa había una tendencia hacia el alza de la presencia de menores en la migración, especialmente asociado con el también incremento de la participación de la mujer en los flujos migratorios. De acuerdo con los datos del Centro de Estudios Migratorios del Instituto Nacional de Migración (сем), los flujos de repatriación de Estados Unidos a México crecieron muy rápidamente desde 1995, cuando llegaron a 853000 repatriaciones, hasta alcanzar más de un millón de eventos de repatriación en el 2000. Pero después vino una disminución, fluctuando entre 525000 y 600000 anuales. Del 2010 en adelante, el promedio varió a 350000 anuales (Centro de Estudios Migratorios del Instituto Nacional de Migración, 2008).

A partir del año 2001, el Instituto Nacional de Migración (INM) comenzó a presentar sus estadísticas de repatriaciones desglosadas por género y edad, y según sus datos, para el periodo 2001-2005 la cantidad de NNA deportados (en promedio anual) alcanzó los 49 387, los cuales representaban $8.47 \%$ del total de personas migrantes repatriadas, incluidas adultas; esto quiere decir que 8 de cada 100 eventos de repatriación se referían a personas menores de 18 años de edad. Del 2006 al 2011 correspondió a 5 de cada 100 eventos de repatriación.

Para 2012 y 2013, según las cifras del InM, continuó el decremento de repatriaciones en general y en consecuencia también el de NNA, pues según se observa en el cuadro 1, en 2012 se registraron 233661 eventos de deportación y de ese total 10697 fueron repatriaciones de NNA; para 2013, las deportaciones totales alcanzaron 33265 eventos, de las cuales 16971 correspondieron a NNA.

Cuando los datos se desagregan según sexo (ver cuadro 2), se observa que dos terceras partes correspondían a NNA varones hasta el 2003, mientras que las nNA mujeres llegaban a un tercio. Pero esa situación fue cambiando en los años posteriores hasta llegar a constituir en el 2013, 8 de cada 10 NNA varones por 2 de cada 10 NNA mujeres.

Los datos del Centro de Estudios Migratorios del INM, 2000-2013, también nos permiten ver cómo la migración tanto de adultos como de NNA 
José A. Moreno Mena y Rosa María Avedaño Millán / Arrinconados por la realidad: Menores de circuito

Cuadro 1. Repatriación de mexicanos desde Estados Unidos.
Adultos y NNA, 2000-2012

\begin{tabular}{|cccc|}
\hline Año & Total general $($ adultos + NNA) & Total $N N A F N$ & Porcentaje $N$ NA \\
\hline 2000 & 1150906 & 116938 & 10.2 \\
2001 & 791256 & 63756 & 8.1 \\
2002 & 583408 & 47585 & 8.2 \\
2003 & 559949 & 52535 & 9.4 \\
2004 & 511028 & 39420 & 7.7 \\
2005 & 536767 & 39910 & 7.4 \\
2006 & 514779 & 37575 & 7.3 \\
2007 & 564609 & 35744 & 6.3 \\
2008 & 559361 & 32151 & 5.7 \\
2009 & 590792 & 25271 & 4.3 \\
2010 & 445877 & 19425 & 4.4 \\
2011 & 396564 & 15167 & 3.8 \\
2012 & 233661 & 10697 & 4.6 \\
2013 & 332865 & 16971 & 5.0 \\
\hline
\end{tabular}

Fuente: Centro de Estudios Migratorios del Instituto Nacional de Migración, 20002013.

se desplazó de un estado a otro, de acuerdo a cómo se presenten los controles fronterizos y los riesgos que conlleva cruzar por lugares cada vez más inhóspitos. A partir del 2000 los eventos de deportación de NNA se desplazaron hacia el estado de Sonora, donde Nogales se convirtió en la ciudad más importante, solamente en el 2007 y 2008 no hegemonizó en las repatriaciones de menores porque Baja California mantuvo la primacía. Para 2012 y 2013 dos estados figuraron como zonas de deportación de NNA, Sonora y Tamaulipas, lo que se reflejó en las estadísticas de atención de los consulados correspondientes a esas entidades (ver cuadro 3).

De la escasa información existente sobre NNA se ha observado que los casos de menores de circuito en los flujos migratorios de NNA, representan una cantidad significativa a la cual debe ponerse atención, ya que hay un riesgo potencial de que este tipo de NNA cada vez más vaya en aumento. Hay algunos ejemplos que pueden ilustrar la cantidad de menores de 
Cuadro 2. Repatriación de niños, niñas y adolescentes, 2000-2012. Porcentajes por sexo

\begin{tabular}{|cccccc|}
\hline Año & Cantidad & \% hombres & Cantidad & \% mujeres & Total \\
\hline 2000 & 75133 & 64.3 & 41805 & 35.7 & 116938 \\
2001 & 42707 & 67.0 & 21049 & 33.0 & 63756 \\
2002 & 32437 & 68.2 & 15148 & 31.8 & 47585 \\
2003 & 33977 & 64.7 & 18558 & 35.3 & 52535 \\
2004 & 28326 & 71.9 & 11094 & 28.1 & 39420 \\
2005 & 29026 & 72.7 & 10884 & 27.3 & 39910 \\
2006 & 27947 & 74.4 & 9652 & 25.7 & 37575 \\
2007 & 26750 & 74.8 & 8994 & 25.2 & 35744 \\
2008 & 24484 & 76.2 & 7667 & 23.8 & 32151 \\
2009 & 19630 & 77.7 & 5641 & 22.3 & 25271 \\
2010 & 15791 & 81.3 & 3634 & 18.7 & 19425 \\
2011 & 12601 & 83.1 & 2571 & 17.0 & 15167 \\
2012 & 9019 & 84.3 & 1679 & 15.7 & 10697 \\
2013 & 14625 & 86.2 & 2346 & 13.8 & 16971 \\
\hline
\end{tabular}

Fuente: Centro de Estudios Migratorios del Instituto Nacional de Migración, 2000-2013.

circuito que cruzan en las fronteras mexicanas con Estados Unidos y que sirven como un referente de las dimensiones que ha tomado el fenómeno. Por ejemplo, el caso de Piedras Negras, Coahuila, el Sistema para el Desarrollo de la Familia Municipal señaló que el fenómeno había disminuido en relación con años anteriores, pero para 2008 registraban en promedio 20 casos al mes de ese tipo de NNA, originarios o residentes de la ciudad, asî como algunos casos que tenían reincidencia de internaciones hacia Estados Unidos de hasta 15 veces; también se manifestó que hasta noviembre de ese año tenían un registro de " 89 jovencitos que oscilan entre las edades de 15 a 17 años de edad, de un total general de 530 menores migrantes que han cruzado hacia Estados Unidos por esta frontera"(Pulgarin, 2010, p. 1). Es decir, $16.5 \%$ de los NNA atendidos por esa dependencia local correspondió a menores de circuito, dato que no es menor, aun considerando que ese fenómeno vaya a la baja en ese municipio fronterizo. 
José A. Moreno Mena y Rosa María Avedaño Millán / Arrinconados por la realidad: Menores de circuito

Cuadro 3. Cantidad de NNA atendidos por la Red Consular de México, según región fronteriza entre Estados Unidos y México, 2012 y 2013

\begin{tabular}{|lrrrr|}
\hline \multirow{2}{*}{ Frontera EUA-México } & \multicolumn{2}{c}{2012} & \multicolumn{2}{c|}{2013} \\
\cline { 2 - 5 } & Absoluto & \multicolumn{1}{c|}{$\%$} & Absoluto & \multicolumn{1}{c|}{$\%$} \\
\hline Texas-Tamaulipas (A) & 4930 & 36.6 & 6880 & 43.0 \\
California-Baja California(B) & 1534 & 11.4 & 1327 & 8.3 \\
Texas-Coahuila (C) & 919 & 6.8 & 1008 & 6.3 \\
Texas-Chihuahua (D) & 657 & 4.9 & 646 & 4.0 \\
Arizona-Sonora (E) & 5414 & 40.3 & 6155 & 38.4 \\
Total & 13454 & 100.0 & 16016 & 100.0 \\
\hline
\end{tabular}

(A) Agrupa datos de los consulados mexicanos ubicados en las ciudades de Brownsville, Laredo y McCallen, ubicados en el estado de Texas; ciudades que a su vez están en la frontera con Matamoros, Nuevo Laredo y Reynosa (respectivamente) en el estado mexicano de Tamaulipas.

(B) Agrupa datos de los consulados mexicanos ubicados en San Diego y Caléxico, California, que son frontera con las ciudades de Tijuana y Mexicali, en el estado mexicano de Baja California.

(C) Incluye datos de las ciudades texanas de Del Río y Eagle Pass, que son frontera, respectivamente, con las ciudades de Ciudad Acuña y Piedras Negras, en la entidad mexicana de Coahuila.

(D) Suma los datos de los consulados mexicanos ubicados en El Paso y Presidio, Texas; que son fronteras, respectivamente, con Ciudad Juárez y Ojinaga, en el estado de Chihuahua. (E) Agrupa los datos de los consulados mexicanos ubicados en Douglas, Nogales y Yuma, Arizona; ciudades cercanas a las fronteras del estado de Sonora, respectivamente: Agua Prieta, Nogales y San Luis Río Colorado.

Fuente: Elaboración propia con base en datos de la Red Consular de México en Fronteras.

Por otro lado, en abril de 2008 la delegación del InM del estado de Chihuahua confirmó la existencia de menores que se dedicaban a colaborar en el tráfico de personas indocumentadas hacia Estados Unidos por la frontera de Ciudad Juárez, a los que se les conocía como "polleritos". En esa ocasión sólo se mencionó la presencia de jovencitos entre los 14 y 17 años de edad que participaban en los cruces o como guías (Chaparro, 2008). Pero en agosto de 2012, esa institución ya tenía detectados a por lo menos 97 menores de edad, que registraron varias entradas no autorizadas a Estados Unidos y regresos frecuentes a México ("Indaga Migración Mexicana”, 2012). 
Por su parte, Hernández (2012), en registros del Centro de Atención para Menores Fronterizos (CAMEF) en Matamoros, encontró que en 2011, $61.2 \%$ de los menores era originario del estado de Tamaulipas, al que pertenece esa ciudad; si bien la cifra no registra la cantidad de NNA originarios o radicados en Matamoros (que serían propiamente los menores de circuito en esa ciudad), el dato resulta importante ya que da una idea de la cantidad de niños y adolescentes originarios de la entidad, que frecuentemente intentan cruzar de manera indocumentada hacia Estados Unidos y están en riesgo de ser cooptados por el crimen organizado.

En cifras de 2013, publicadas por el Observatorio de Legislación y Política Migratoria de El Colegio de la Frontera Norte, encontramos que del total de niños, niñas y adolescentes repatriados a México, 4981 eran originarios de alguno de los seis estados mexicanos fronterizos que colindan con Estados Unidos; de ese total de la frontera norte, $5.6 \%$ estaba acompañado de algún adulto y $94.4 \%$ correspondió a NNA que viajaban solos al momento de ser detenidos por la patrulla fronteriza (ver cuadro 4).

Estos datos proporcionan una dimensión "más real" de la cantidad de nNa provenientes de las entidades fronterizas del norte de México que

Cuadro 4. Niñas, niños y adolescentes repatriados de Estados Unidos, originarios de los estados fronterizos del norte de México, 2013

\begin{tabular}{|c|c|c|c|c|c|c|c|c|c|c|c|c|c|c|}
\hline & \multicolumn{2}{|c|}{$\begin{array}{c}\text { Baja } \\
\text { California }\end{array}$} & \multicolumn{2}{|c|}{ Sonora } & \multicolumn{2}{|c|}{ Chihuahua } & \multicolumn{2}{|c|}{ Coahuila } & \multicolumn{2}{|c|}{$\begin{array}{c}\text { Nuevo } \\
\text { León }\end{array}$} & \multicolumn{2}{|c|}{ Tamaulipas } & \multicolumn{2}{|c|}{ Total F. N. } \\
\hline & $A b s$ & $\%$ & $A b s$ & $\%$ & $A b s$ & $\%$ & $A b s$ & $\%$ & $A b s$ & $\%$ & $A b s$ & $\%$ & $A b s$ & $\%$ \\
\hline \multicolumn{15}{|l|}{ Acompañados } \\
\hline 12 a 17 años & 24 & 0.5 & 12 & 0.2 & 36 & 0.7 & 15 & 0.3 & 28 & 0.6 & 70 & 1.4 & 185 & 3.7 \\
\hline Hasta 11 años & 17 & 0.3 & 3 & 0.1 & 25 & 0.5 & 10 & 0.2 & 18 & 0.4 & 19 & 0.4 & 92 & 1.8 \\
\hline Subtotal & 41 & 0.8 & 15 & 0.3 & 61 & 1.2 & 25 & 0.5 & 46 & 0.9 & 89 & 1.8 & 277 & 5.6 \\
\hline \multicolumn{15}{|c|}{ No acompañados } \\
\hline 12 a 17 años & 249 & 5.0 & 2343 & 47.0 & 468 & 9.4 & 350 & 7.0 & 69 & 1.4 & 1188 & 23.9 & 4667 & 93.7 \\
\hline Hasta 11 años & 4 & 0.1 & 5 & 0.1 & 3 & 0.1 & 3 & 0.1 & 1 & 0.02 & 21 & 0.4 & 37 & 0.7 \\
\hline Subtotal & 253 & 5.1 & 2348 & 47.1 & 471 & 9.5 & 353 & 7.1 & 70 & 1.4 & 1209 & 24.3 & 4704 & 94.4 \\
\hline Total & 294 & 5.9 & 2363 & 47.4 & 532 & 10.7 & 378 & 7.6 & 116 & 2.3 & 1298 & 26.1 & 4981 & 100.0 \\
\hline
\end{tabular}

Fuente: Realizado a partir de datos del Observatorio de Legislación y Política Migratoria-El colef (2014). Mapa de rangos de menores repatriados por Estados Unidos según entidad de origen durante 2013. 
están cruzando de manera indocumentada hacia Estados Unidos, pues su residencia cercana a la franja fronteriza les facilita realizar cruces recurrentes, lo que potencialmente puede convertirlos en menores de circuito. En el cuadro 4 puede observarse que los estados fronterizos del norte mexicano que "aportaron" más NNA repatriados en 2013, fueron Sonora $(47.1 \%)$ y Tamaulipas $(24.3 \%)$, ya que en conjunto $71.4 \%$ de los NNA provenientes de la frontera norte eran originarios de esas dos entidades.

Para visualizar mejor a los menores de circuito en las estadísticas sobre NNA en general, es necesario recurrir al reactivo motivaciones migratorias para intentar cruzar de manera no autorizada hacia Estados Unidos. Esto resulta importante para entender el contexto en que se ven inmersos los menores de circuito, pues como la mayoría de los otros NNA, buena parte de ellos también ha emprendido su trayecto migratorio en busca de oportunidades de empleo para mejorar su situación económica, y al ver frustradas sus esperanzas y como una forma de sobrevivencia, se han involucrado en actividades ilícitas. De ahí que resulte relevante destacar que, desde hace algunos años, se ha documentado que la migración de NNA hacia Estados Unidos dejó de tener como motivación principal la reunificación familiar y actualmente tiene su base más importante en necesidades económicas y laborales.

En la Red Consular perteneciente a la Dirección General de Protección a Mexicanos en el Exterior de la Secretaría de Relaciones Exteriores (SRE) se realizó una exploración estadística del periodo 2012 y 2013, a partir de la pregunta motivaciones principales para migrar a NNA atendidos. En los resultados incluidos en el cuadro 5, se puede observar que de los 13454 NNA atendidos por la Red Consular en el año 2012, 69.1\% manifestó que sus motivaciones eran de tipo laboral, mientras que sólo $10.1 \%$ indicó la reunificación familiar.

En el año 2013, de un total de 16016 nNa, lo laboral para haber realizado el cruce no autorizado fue lo que motivó al 58.8\% y la de reunirse con la familia alcanzó 9.6\%. En ambos casos los porcentajes disminuyeron en relación con el año anterior, sobre todo la motivación laboral, que bajó más de 10 puntos porcentuales; pero aun así, buscar trabajo y reunirse con familia continuaron siendo las dos razones principales para intentar cruzar hacia Estados Unidos. 
Cuadro 5. Principal motivo para la migración manifestado por NNA, 2012

\begin{tabular}{|lrrrr|}
\hline \multicolumn{1}{r}{$\begin{array}{c}\text { Principal motivo } \\
\text { para cruzar }\end{array}$} & \multicolumn{3}{c}{2012} & \multicolumn{1}{c|}{2013} \\
\cline { 2 - 5 } & Cantidad & \multicolumn{1}{c}{ Cantidad } & \multicolumn{1}{c|}{$\%$} \\
\hline Laboral & 9295 & 69.1 & 9421 & 58.8 \\
Reunificación familiar & 1355 & 10.1 & 1533 & 9.6 \\
Estudios & 671 & 5.0 & 747 & 4.7 \\
Visita & 157 & 1.2 & 86 & 0.5 \\
Vagancia & 881 & 6.5 & 2157 & 13.5 \\
Turismo & 61 & 0.5 & 64 & 0.4 \\
Acompañamiento a un adulto & 42 & 0.3 & 52 & 0.3 \\
Radica en EUA & 36 & 0.3 & 7 & 0.0 \\
Tráfico de personas & 514 & 3.8 & 1172 & 7.3 \\
Tráfico de drogas & 190 & 1.4 & 290 & 1.8 \\
Otro & 189 & 1.4 & 421 & 2.6 \\
No disponible & 63 & 0.5 & 66 & 0.4 \\
Total & 13454 & 100.0 & 16016 & 100.0 \\
\hline
\end{tabular}

Fuente: Elaborado a partir de información de la Red Consular de la Dirección General de Protección a Mexicanos en el Exterior de la Secretaría de Relaciones Exteriores (2013).

Pero las cifras más contundentes que permiten señalar que el fenómeno de los menores de circuito va en aumento se pueden observar en la figura 1, en el reactivo de la motivación principal, donde del total de los 13454 NNA detenidos y repatriados desde EUA, 6.5\%, manifestó "la vagancia" para haber cruzado hacia el vecino país. Esa misma razón aumentó casi al doble en el 2013 con 2157 NNA, representando 13.5\% del total.

Adicionalmente, si sumamos los reactivos tráfico de personas y de drogas del 2012, se tiene que 5.2\% (704) de los niños, niñas y adolescentes no acompañados reconoció que cruzaron a territorio estadunidense por esas razones; pero para 2013, los que manifestaron esos mismos motivos sumaron $9.1 \%$ de los NNA. Cuando agrupamos los reactivos de vagancia, tráfico de personas y tráfico de drogas encontramos que en el 2012 suman 11.7\% del total de NNA registrados por la Red Consular, pero el año siguiente, es 


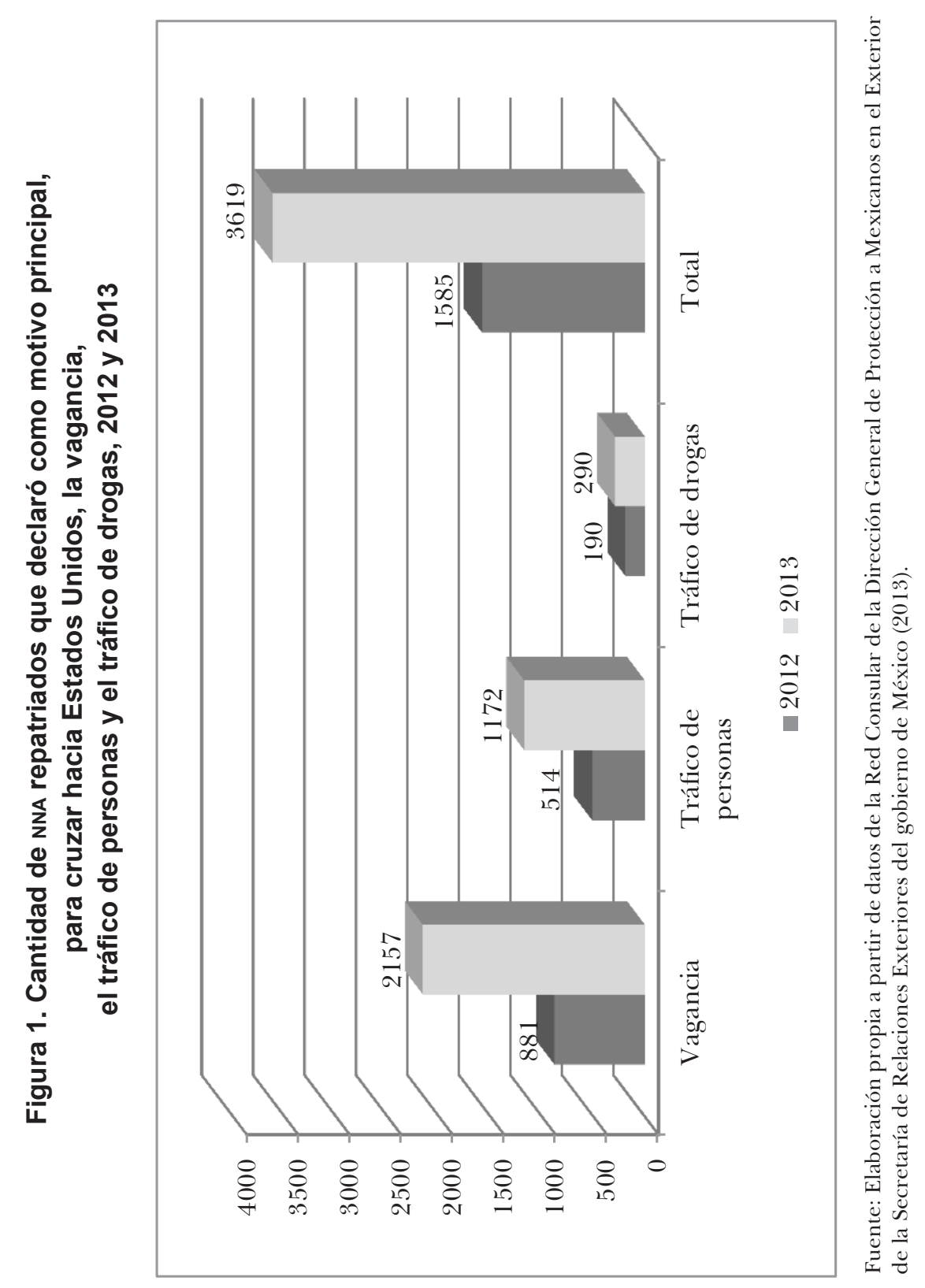


decir en 2013, ascendieron a 22.6\% del total de NNA repatriados. Las cifras muestran que sólo en un año aumentó de manera exponencial la cantidad de niños, niñas y adolescentes no acompañados que cruzaron hacia Estados Unidos y que podrían caracterizarse como menores de circuito. La información no sólo ilustra que el fenómeno va en aumento, sino que nos encontramos frente a una situación preocupante que requiere de manera urgente políticas públicas dirigidas a impedir que los NNA migrantes sigan exponiéndose o engrosando las filas de los menores de circuito.

\section{Situación de riesgo y vulnerabilidad para NNA en la frontera norte}

En la frontera norte de México los migrantes de distintos perfiles, los que llegan con la intención de cruzar hacia Estados Unidos, los deportados y los centroamericanos, se encuentran con el muro virtual y físico que se ha venido construyendo a partir de la famosa operación de control migratorio "Operación Guardián", iniciada en la primera mitad de la década de los noventa en el gobierno de Clinton y extendida con la política de securitización de los presidentes que le precedieron, motivados por los acontecimientos del 11 de septiembre de 2001, con la acción terrorista del derribo de las Torres Gemelas en Nueva York. Esta nueva visión que se impuso en los gobiernos estadunidenses, pone a la migración junto con el terrorismo y el narcotráfico como asuntos de seguridad nacional (Moreno, 2013). Lo anterior, aunado a la guerra desarrollada por el gobierno anterior contra el narcotráfico y el crimen organizado, y que no ha cambiado mucho con el actual gobierno, generó que las regiones por las que transitaban regularmente las personas migrantes se encontraran en estado de extrema violencia e inseguridad. Se puede afirmar que se ha institucionalizado un contexto de riesgo donde los más vulnerables son los niños (Bustamante, 2001; Ruiz, 2004).

En la frontera norte de México los migrantes convergen con similares necesidades en un espacio por demás hostil. Están atrapados en un terreno complejo donde interactúan criminales de tráfico y trata, "polleros, narcotraficantes y bajadores de migrantes" que pretenden extorsionarlos, secuestrarlos y hasta asesinarlos, como ha sido el caso de la masacre de 72 
personas en agosto de 2010 y el descubrimiento posterior de fosas clandestinas en San Fernando, Tamaulipas. A estos grupos de delincuentes se agregan las policías locales que se aprovechan de la vulnerabilidad de los migrantes y que a menudo actúan en complicidad con las bandas de criminales. En la actualidad los migrantes se encuentran entre dos fuegos: por un lado, frente a las violaciones a los derechos humanos de la Patrulla Fronteriza, policías locales y el Ejército Mexicano; y por otro, frente al crimen organizado, que vio en los migrantes una preciada mercancía (Moreno, 2013). Es en estos entornos de conflictivad intrafronteriza, donde los NNA presentan los más altos niveles de vulnerabilidad, donde además tienen que enfrentarse:

[...] A un mundo que altera severamente las posibilidades de desarrollo de todas sus potencialidades, y los expone a situaciones que llegan a dañarlos gravemente. Estas situaciones son el abandono familiar, la explotación laboral o sexual, su alejamiento de las instituciones educativas o involucrarse con grupos de riesgo que los empujan en una espiral de daños cada vez mayores (Petit, 2003, p. 17).

En ese escenario los NNA son los más susceptibles a la violación de sus derechos, dado que viajan solos o bien quienes los acompañan no necesariamente son las personas más adecuadas para su protección. Por esa simple razón se ven expuestos a situaciones de alto riesgo, en las cuales su integridad física se encuentra indefensa ante las redes del tráfico de personas, narcotraficantes y de trata sexual. Al respecto, Ramírez et al. (2009) advierten que aún hay un sinnúmero de riesgos a la integridad física de los NNA repatriados y en tránsito en la línea fronteriza, tales como: el riesgo de caer en manos de traficantes de personas; abusos, maltrato y discriminación; el no respetar los horarios convenidos en los arreglos locales y memorándum de repatriación; el no contar con los espacios adecuados para brindarles atención. Asimismo, la violación al derecho de información cuando sus padres son detenidos y ellos no se enteran y no son informados sobre su paradero. También es frecuente el secuestro de migrantes en la frontera norte, y los NNA no son la excepción. Ya anteriormente, en el estudio de Gallo (2004), se advertía que en la frontera norte: 
Uno de los grupos más susceptibles a la violación de sus derechos son los niños, niñas y adolescentes migrantes que cruzan la frontera o que intentan cruzarla de manera irregular sin la compañía de familiares adultos [...] además de la búsqueda de una mejor forma de vida, estos niños y niñas generalmente cruzan la frontera con el anhelo de reunirse con sus familiares que ya se encuentran en Estados Unidos [...] en esos desplazamientos pueden ser involucrados en la comisión de delitos, ser enganchados en redes de explotación sexual o laboral, pueden sufrir accidentes e incluso morir (p. 10).

En su segundo informe de 2013, el PDIB también menciona otros tipos de riesgos que corren los NnA producto de los procesos de repatriación; uno de ellos es la separación familiar, que atenta contra el derecho a la unidad familiar. El otro es la separación por medio de las repatriaciones laterales. Éstas atentan contra la integridad y seguridad personal de los NNA, aumentando el riesgo de ser colocados en fronteras inseguras y zonas desconocidas, principalmente por los estados del noreste mexicano como Coahuila y Tamaulipas (Programa de Defensa e Incidencia Binacional, 2013a).

Aludiendo a la Patrulla Fronteriza de Estados Unidos, Pérez señala:

Muchos de los NNA detenidos en la frontera se arriesgaron a viajes peligrosos hacia Estados Unidos, buscando una mejor oportunidad de vida, económicas y de educación. Algunos migraron para escapar de circunstancias intolerables en sus hogares y, para el momento en el que atraviesan la frontera, muchos llegan a Estados Unidos habiendo sido víctimas de trata o manipulados por los grupos criminales en México (Pérez, 2012, p.12).

En ese contexto, los NNA de circuito se encuentran en una condición todavía más vulnerable, pues se han convertido en carne de cañón. Son reclutados por dinero o simplemente son obligados bajo amenazas a cruzar la frontera guiando a personas o llevando remesas de droga, como lo refiere el coordinador general de las Casas YMCA para menores migrantes Uriel González:

Además de ser utilizados como guías, los jóvenes que cruzan la frontera por la zona montañosa de Baja California corren el riesgo de ser cooptados por los 
José A. Moreno Mena y Rosa María Avedaño Millán / Arrinconados por la realidad: Menores de circuito

cárteles de la droga mexicanos. Están pagando un peaje, no en efectivo sino en especie. Los grupos de tráfico de drogas están obligando a los migrantes, incluyendo menores de edad, a ser portadores de la droga hacia los EU. Eso inmediatamente cambia el propósito y la penalidad de ingreso. Dejan de ser un migrante que entra de manera irregular a un país y se convierten en un traficante de drogas (Agencia Reforma, 2009, párr. 6).

La ventaja de las bandas del crimen organizado al enganchar a este segmento poblacional de la niñez migrante, es que estos NNA no son sujetos a un proceso criminal por su actividad de guías de personas o de trasiego de droga cuando son detenidos en territorio norteamericano y sólo son repatriados a México (Programa de Defensa e Incidencia Binacional, 2012).

Esta situación ya se había hecho notar en algunos foros, como ocurrió en el seminario Niñez Migrante en 2006, donde se advirtió de la existencia de una:

[...] práctica que permite que el menor sea devuelto por las autoridades inmigratorias al intentar entrar a los Estados Unidos en violación a sus leyes de inmigración, infinidad de veces sin que sea detenido de manera permanente.

La aplicación de esta medida por sí sola, ha generado un fenómeno social inesperado: los menores al gozar de una especie de inmunidad fronteriza han sido utilizados como una herramienta más de la industria de la migración informal o ilegal; los niños y adolescentes son usados dentro de la estructura de las bandas del crimen organizado como traficantes de personas o de mercancías prohibidas (El Colef, 2006, p. 9).

En algunos casos, estos NNA de circuito son utilizados como testigos de cargo, dentro de investigaciones del orden penal por tráfico de personas y/o droga cuando son detenidos en Estados Unidos; sin embargo, una vez concluida su participación en dichos procesos, son repatriados a México, hecho que pone en riesgo su vida misma porque pueden ser víctimas de represalias por parte de los miembros de las redes criminales con las que estaban involucrados/as, transgrediendo así el principio de no devolución en relación con los niños y niñas migrantes. 


\section{Vulnerabilidad social de los menores de circuito}

Como se puede observar, los riesgos que corren los NNA en la frontera son objetivos, desde los propiciados por el clima, los desiertos y ríos, hasta los propiciados por otros seres humanos. Aquí conviene hacer un paréntesis para abordar la vulnerabilidad social que viven los menores de circuito. $\mathrm{Al}$ principio mencionamos que estos niños se encuentran inmersos en una realidad social que los arrincona, que impide su desarrollo. Ignorados por un estado que no propicia las oportunidades, y que no tiene políticas de prevención, mucho menos de protección. Por eso tenemos que dar una mirada al entorno familiar de estos menores.

No hay estudios que nos hablen del ámbito familiar de los menores de circuito, sin embargo, por la experiencia de encargados de los albergues de menores migrantes (González, 2014), nos podemos dar cuenta de la complejidad del asunto, en algunos casos las familias de los menores de circuito están separadas por la línea que divide a México de Estados Unidos y la figura paterna está ausente. La organización parental casi siempre está centrada en la figura materna. Pero se da también el caso contrario, donde el padre y los hijos fueron deportados, mientras que la madre se quedó en Estados Unidos.

En otros casos, también la madre está "ausente" por motivos de trabajo, salud u abandono. En otro escenario, la familia de origen está en constante conflicto y la violencia intrafamiliar es algo cotidiano, por lo que retomando a Scandizzo (citado en Bulgach y Flores, 2011) los menores sufren un proceso de "autoexpulsión condicionada", o en otras palabras, "un intento por parte del niño por resolver el estado de tensión que plantea la permanencia en su hogar" (p. 307). Este proceso no es rápido sino gradual, en donde el niño va adoptando el ámbito fuera del hogar como espacio de socialización y de subsistencia. Este niño entra en la subcategoría de los niños separados de sus familias que hace unicEF de "niño en peligro"; "que viven en casa pero son susceptibles de huir de ella" (Elizaguirre, 2006, párr. 2).

Pero también se presentan entornos familiares relativamente integrados con padre y madre con graves carencias económicas, donde los niños comienzan a elaborar estrategias de vida que los llevan a automatizarse del sustento económico familiar preponderante y los proveen de cierto estatus 
José A. Moreno Mena y Rosa María Avedaño Millán / Arrinconados por la realidad: Menores de circuito

dentro del grupo familiar (Oropeza, 2014). Según lo refiere la Red de Casas YMCA, los menores de circuito, en algunos casos, destinan sus ingresos al sustento familiar y para sufragar sus propios gastos:

[...] en nuestras organizaciones se tuvo el caso de un niño repatriado al cual se le brindó la atención correspondiente y que al hablar con él se supo de su actividad como guía; posteriormente, se pudo establecer contacto con su madre para llevar a cabo la reunificación familiar y al platicar con ella sobre el gran riesgo que representaba para el niño dicha actividad, ésta manifestó que lo entendía; sin embargo, el niño aportaba a la casa 500 dólares por semana y no podían prescindir de ese ingreso (Programa de Defensa e Incidencia Binacional, 2012, p.11).

En relación con lo anterior, Pérez menciona que en el tema de los NNA de circuito también se presenta otro fenómeno: el de padres que utilizan a sus hijos para servir de "polleros" con otros menores, debido a que el papá recibe por el cruce de cada menor entre 300 y 500 pesos (Pérez, 2012). En esos casos los adultos promueven que los niños se involucren en estrategias de carácter paliativo, con el propósito de ampliar la fuente de ingresos alternativos; por estas situaciones, las actividades de los menores de circuito también se han convertido en parte de su cultura de supervivencia, ya que estos NNA, al no tener más opciones para sobrevivir, han sido cooptados por bandas criminales y se dedican a cruzar en repetidas ocasiones hacia Estados Unidos, para realizar diversas actividades para los grupos a los que ahora pertenecen.

Si las situaciones familiares precarias orillan a los menores a buscar recursos monetarios para sufragar sus necesidades y las de su familia, la desatención de las autoridades agrava el problema. Algunos servidores públicos del lado mexicano, se han atrevido a reconocer que tanto para las instituciones de migración como para las encargadas de dar albergue a los menores de circuito, la situación es grave. Al respecto, la Oficial de Protección a la Infancia (OPI) del Instituto Nacional de Migración (INM), Patricia Fragoso, ha señalado que "es un tema que requiere de una negociación bilateral, porque tanto los oficiales estadunidenses detectan quién ha cruzado, por dónde y cuántas veces, y aun cuando saben que es 
el mismo, y que se identifica con diversos nombres en cada detención, no nos informan" (Pérez, 2012, p.12).

El tema también se ha complejizado por los distintos enfoques de las autoridades, tanto mexicanas como estadunidenses, como lo ha advertido Appleseed (2011), pues mientras que el gobierno mexicano, por medio del Instituto Nacional de Migración, se ha empeñado en retener a los NNA para evaluar las condiciones por las que decidieron migrar y los riesgos que representa la reinserción familiar, el DIF opta por apresurar la reunificación en lugar de realizar una evaluación profunda de los motivos del menor para cruzar la frontera, así como una evaluación del ambiente del hogar del mismo (Appleseed, 2011). Pero por otro lado, el enfoque estadunidense prioriza la devolución a su país de origen sin investigar si han sido victimizados en sus lugares de origen, si han sido detenidos, sin atender al derecho superior del niño. Simplemente los devuelven, como sostiene el estudio de Appleseed (2011). Por todo ello, los niños, niñas y adolescentes no acompañados, que han sido detenidos en el intento de cruzar las fronteras de México con Estados Unidos, y que son deportados sin considerar los peligros que enfrentan al ser devueltos sin medidas de protección y procedimientos adecuados, se encuentran en grave riesgo.

El asunto de los menores de circuito es que deberían ser vistos con un enfoque transversal, porque involucra a los tres niveles de gobierno; por su complejidad, las autoridades no han definido cómo asumir sus responsabilidades, sobre todo de alejar a los niños de las redes de tráfico y garantizarles un ambiente libre de violencia, como establecen los derechos del niño.

En una entrevista sobre el tema de los menores traficantes, Moreno García $^{7}$ menciona que eso causó escozor entre las distintas dependencias ya mencionadas:

Migración tuvo mucho tiempo la papa caliente en las manos. Por un lado estaba EU presionando porque no teníamos ningún protocolo de acción. Por el otro lado, el DIF que ya no me los aceptaba. Asimismo, las autoridades migratorias de EU informan al consulado antes que a nosotros. Lamentablemente creo que nadie le quiso entrar a los toros por los cuernos (Gutiérrez, 2012, párr. 25).

${ }^{7}$ Ex subdirectora de Protección al Migrante del InM en Baja California, de 2003 a 2010 . 
José A. Moreno Mena y Rosa María Avedaño Millán / Arrinconados por la realidad: Menores de circuito

De igual manera, en el sistema consular mexicano no hay claridad de qué autoridad debe hacerse responsable de atender el problema:

La cónsul mexicana Remedios Gómez, ubicada en San Diego, California, una de las principales entradas a EU que emplean los migrantes indocumentados, así como de punto de recepción de las deportaciones, puntualizó que el problema de los menores guía sale de las atribuciones de su oficina: "No es competencia del consulado investigar quiénes son los menores guías[...] Las autoridades estadunidenses tienen este récord [de quiénes son los reincidentes][...] No es facultad de nosotros estar buscando esa información (Gutiérrez, 2012, párr. 27).

Lo que es peor, en el caso de los menores de circuito, a pesar del riesgo en México, la principal institución encargada de protegerles, atenderles, resguardarles y buscarles oportunidades para una reintegración adecuada y segura a sus senos familiares o a la sociedad, omite su responsabilidad e incluso se ha negado a recibir a estos menores en sus albergues, tal como lo señaló una opI en un medio de comunicación:

El Sistema Nacional para el Desarrollo Integral de la Familia (DIF) nacional nos ha dicho que no quiere recibir a estos menores repatriados, porque ponen en peligro a su personal y a otros niños que están en el refugio. Hay ocasiones en que reciben a ese menor y en pocas horas se presenta un tipo bien violento que, acompañado de un grupo que rodea el albergue, exige llevárselo, identificándose como su familiar y tienen que dejarlo ir (Pérez, 2012, p.12).

El Sistema Nacional Dif tampoco se ha preocupado por establecer una política de atención que proteja y proporcione mayor seguridad a los menores de circuito. Lo anterior pudo constarse a través de la respuesta dada por el sistema federal DIF a una solicitud de información que se le hizo a través del Instituto Federal de Acceso a la Información (IFAI) en julio de 2013, a la que se respondió en los términos siguientes:

La informacion solicitada, es inexistente en los archivos de esta Dirección General, toda vez que, conforme a lo dispuesto por el artículo 29 del Estatuto 
Orgánico del Sistema Nacional del Desarrollo Integral de la Familia, las facultades que tiene encomendadas, entre otras, son las de establecer, promover y ejecutar políticas y lineamientos así como programas y proyectos en materia de prevención y atención de riesgos sociales, así como problemáticas que afecten a la infancia y adolescencia en situación de vulnerabilidad, promover estudios e investigaciones sobre causas y efectos de la vulnerabilidad social que afecta a la infancia y adolescencia; e instrumentar programas y acciones del Organismo, orientados a la prevención de riesgos que enfrenten las niñas y niños en edad temprana, en énfasis en aspectos asistenciales y formativos. En este contexto, y en consecuencia, esta Unidad Administrativa, conforme a la normatividad vigente no está obligada a contar con la información solicitada, y por lo tanto, resulta imposible que la proporcione (Programa de Defensa e Incidencia Binacional, 2013b, p. 1).

Hay pues una grave omisión, no sólo por la ausencia de información acerca de los menores de circuito repatriados o atendidos en albergues, sino sobre todo porque el sistema federal para el desarrollo de la familia evade su responsabilidad como institución protectora de la niñez mexicana, para instrumentar programas y acciones orientados a la prevención de riesgos que enfrenten las niñas y niños en edad temprana. Como si el hecho de que estos NNA se involucren en actividades ilícitas y puedan ser cooptados por bandas criminales no significara un riesgo social y no constituyera una situación de vulnerabilidad social que afecta a la infancia y adolescencia en México, tal como lo dejan claro en la respuesta dada a la organización civil PDIB.

\section{Conclusiones}

Como se pudo constatar, los menores de circuito aparecen en los estudios de NNA casi invisibles, sin embargo, son identificados indirectamente en las tipologías que realizan los autores. En las estadísticas oficiales no son registrados correctamente y pasan desapercibidos en una generalidad que impide diseñar políticas públicas específicas para este sector. Aunque constituyen un segmento de NNA, las instituciones encargadas de atender al público infantil los desdeñan y omiten atender su situación; 
sin embargo, se tiene que reconocer su existencia así como sus problemas particulares.

En este trabajo nos propusimos visibilizar el fenómeno y para ello recurrimos a fuentes directas como entrevistas y a fuentes secundarias en registros estadísticos oficiales. En estas últimas, la especificidad de los menores de circuito se ha construido tomando algunos reactivos de entrevistas realizadas por la red consular mexicana en Estados Unidos, constatando un incremento importante en este tipo de menores.

Concluimos también que por su situación de estar en la calle y su vinculación con distintos actores relacionados con el entorno de violencia en la frontera, los menores de circuito deben ser tipificados como un sector en riesgo. Estos menores, antes de ser vistos como criminales, deben ser considerados víctimas puesto que son explotados y orillados a vivir en la clandestinidad; además de que están acumulando antecedentes en los archivos de las autoridades estadounidenses y mexicanas, los que en un futuro, cuando cumplan la mayoría de edad, podrán ser utilizados para fincarles penas más prolongadas al momento de ser detenidos. Como población en riesgo social permanente, deben tener un tratamiento especial, y es ahí donde el Estado mexicano tiene que intervenir. Los sistemas estatales DIF, en coordinación con el federal, deben asumir su responsabilidad en el tema y no evadir a los menores de circuito.

Los menores de circuito pasan por las manos del Instituto Nacional de Migración, de los sistemas para el desarrollo de la familia estatales, de los consulados mexicanos en Estados Unidos, así como por las autoridades del país vecino, sin que, a la fecha, se hayan puesto de acuerdo en cómo proceder para evitar que una vez que han sido detectados en Estados Unidos y repatriados a México, ya no se regresen al círculo de violencia y delincuencia. Se requiere un enfoque en derechos humanos al tratamiento recibido por estos niños, niñas y adolescentes migrantes.

Asimismo, esta población infantil, al abandonar sus estudios y dedicarse a actividades ilícitas, es condenada a perder la oportunidad de un trabajo y vida dignos para el futuro. Desafortunadamente, su situación se hace más compleja cuando la propia familia interviene, por necesidad económica, y se convierte en parte del problema al avalar las acciones de los menores. Por lo tanto, se debe trabajar con las familias de estos menores, 
conocer su situación económica y establecer programas que ayuden a sustituir el ingreso que detenta de las actividades ilícitas.

Se deben revisar los marcos actuales de protección a los niños migrantes de los consulados, el Instituto Nacional de Migración y el sistema federal Dif. Es necesario también que las instancias que reciben y desarrollan todo el proceso de revinculación de los niños migrantes establezcan un protocolo de actuación para los menores de circuito, a fin de velar por el interés supremo del menor.

Por un lado, el Estado mexicano debe realizar políticas públicas enfocadas a proteger a ese sector vulnerable de NNA, a partir de las cuales se priorice el apoyo a las familias de los menores de circuito para que éstos tengan la oportunidad de estudiar y desarrollarse en ambientes más sanos y armónicos que les posibilite alejarse de las organizaciones criminales. Por otro lado, debe castigarse con todo el rigor de la ley a quienes utilicen como instrumentos de sus crímenes a NNA, cuidando siempre de no victimizar a las familias y los menores.

\section{Bibliografía}

Academia Mexicana de Derechos Humanos, Albergue del Desierto y Centro de Apoyo al Trabajador Migrante (1998). La esperanza truncada. Menores deportados por la garita Mexicali-Caléxico. Mexicali: Autor.

Agencia Reforma (2009). Reclutan "polleros” a adolescentes. El Siglo de Torreón. 6 de septiembre, Recuperado de: <http://www.elsiglodetorreon.com.mx/noticia/ 458822.html>

Alto Comisionado de las Naciones Unidas para los Derechos Humanos (1989). Convención sobre los Derechos del Niño. Recuperado de: <http://www2.ohchr. $\mathrm{org} / \mathrm{spanish} / \mathrm{law} / \mathrm{crc} . \mathrm{htm}>$

Appleseed. (2011). Niños en la frontera: Evaluación, protección y repatriación de niños, niñas y adolescentes mexicanos sin compañía. Recuperado de: <http://www.unicef. org/mexico/spanish/Reporte_Ninos_en_la_Frontera_(Espanol).pdf $>$

Autoridades rescatan a 61 migrantes secuestrados en el norte de México (8 de noviembre de 2010). Reuters, últimas noticias. Recuperado de: <http:// mx.reuters.com/article/topNews/idMXL2N0IT1RT20131108>

Bulgach G. y Flores M. (2011). Niñez y adolescencia en situación de calle. Un análisis transversal desde el género. En A. J. Arias, G. B. García y A. Bazzalo (Comps.), IV Encuentro Internacional de Políticas Públicas y Trabajo Social. 
José A. Moreno Mena y Rosa María Avedaño Millán / Arrinconados por la realidad: Menores de circuito

Aportes para la Reconstrucción de lo Público. Carrera Trabajo Social (pp. 305-325). Recuperado de: <http://trabajosocial.sociales.uba.ar/jornadas/contenidos/home. htm>

Bustamante, J. (2001). Un marco conceptual de referencia acerca de la vulnerabilidad de los Migrantes como sujetos de los derechos humanos. En T. Fernández (Coord.), Los rostros de la violencia (pp. 19-53). Tijuana: El Colegio de la Frontera Norte.

Cabrera, D. A. y Valdéz-Gardea, G. C. (2013). La niñez migrante: Un sector que demanda mayor atención pública. En G. Cordova, J. Dutrim, E. Lara y J. G. Rodríguez (Coords.), Desarrollo humano transfronterizo: Retos y oportunidades en la región Sonora-Arizona (pp. 349-369). Sonora: El Colegio de Sonora, El Colegio de la Frontera Norte, Universidad de Sonora.

Centro de Estudios Migratorios del Instituto Nacional de Migración. (2008). Repatriación de mexicanos de Estados Unidos, cuadro 5. Recuperado de: <http:// www.gobernacion.gob.mx/es_mx/SEGOB/Repatriacion_de_mexicanos_2008>

Chaparro, A. (2008). Desmienten al INM, no "hay niños polleritos". Organización Editorial Mexicana, 18 abril. Recuperado de: <http://www.oem.com.mx/esto/ notas/n667757.htm>

Desarrollo Integral para la Familia, Sonora (DIf-Sonora). (2013). Procedimientos para atención a niñas, niños y adolescentes migrantes, repatriados no acompañados, 30 de Agosto. Recuperado de: <http://transparencia.esonora.gob.mx/NR/ rdonlyres/D9A57471-CBB0-4E4A-B95D-7A25BA7D1CD5/99606/64APVP14 Atenci\%C3\%B3naMenoresMigrantes 1.pdf>

Desarrollo Integral para la Familia, Tamaulipas. (2013). Operatividad y normatividad 2013-2016. Recuperado de: <http://www.gomezfarias.gob.mx/wp-content/ uploads/2013/12/MANUAL-DE-OPERATIVIDAD-CAMEF-2013-v2.pdf>

El Colegio de la Frontera Norte (2006). Memoria del Seminario Niñez migrante no acompañada en la frontera norte: Repatriación, protección y atención. Tijuana: El Colef, Unicef, Coalipro, Sin Fronteras, 6 de junio.

Elizaguirre, M. (2006). Niños de la calle. En C. Pérez de Armiño (Ed.), Diccionario de acción humanitaria y cooperación al desarrollo. Recuperado de: <http://www. dicc.hegoa.ehu.es/listar/mostrar/155>

Gallo, K. I. (2004). Niñez migrante en la frontera norte: Legislación y procesos. México: DIF, Unicef.

González, U. (2014). Entrevista a Uriel González/Entrevistador: J. A. Mena, 25 de abril. Copia en posesión del autor.

Gutiérrez, M. G. (26 de noviembre de 2012) No son niños "polleros”, son víctimas. Animal político. Recuperado de: <http://www.animalpolitico.com/2012/11/noson-ninos-polleros-son-victimas/\#axzz32QXhW43P>

Hernández, O. M. (2012). Migración, masculinidad y menores repatriados en la frontera Matamoros-Brownsville. Trayectorias, 33-34(14), 76-94. 
Indaga Migración mexicana nexos de menores con red de "polleros". (18 de agosto de 2012). Noticias la Información E el Acontecer en Camargo, Chihuahua. Recuperado de: <http://internetchihuahua.com/lg2/?p=4831>

Le Goff, H. (2012). Niñas, niños y adolescentes migrantes extranjeros no acompañados en México. Rayuela: Revista Iberoamericana sobre Niñez y Juventud en Lucha por sus Derechos, (5), 129.

López, G. (Coord.) (2003). Diáspora michoacana. Zamora, Michoacán: Gobierno del Estado de Michoacán, El Colegio de Michoacán.

López, G. y Villaseñor, B. (2001). Los menores en el flujo de migrantes. Migración: México entre sus dos fronteras (pp. 115-123). México: Foro Migraciones, Sin Fronteras.

Mancillas, C. (2009). Migración de menores mexicanos a Estados Unidos. En P. Leite y E. Giorguli (Coords.), El estado de la migración. Las políticas públicas ante los retos de la migración mexicana a Estados Unidos. México: Conapo.

Méndez, J. (2000). Presencia de menores en la migración internacional: Un estudio exploratorio del perfil sociodemográfico de los menores repatriados por la Ciudad de Tijuana, 1999, Tesis de maestría. Tijuana, México, El Colegio de la Frontera Norte.

Moreno, J. A. (1 de septiembre de 2013). Bienvenidos al infierno. México Social, (38), 26-29. Recuperado de: <http://mexicosocial.org/index.php/secciones/ especial/item/342-bienvenidos-al-infierno>

Nexos (12 de enero de 2012). ¿¿ué es una opinión Amicus Curaie? [Mensaje de un blog]. Recuperado de: <http://eljuegodelacorte.nexos.com.mx/?p=1630>

Observatorio de Legislación y Política Migratoria, El Colegio de la Frontera Norte (2014). Mapa de rangos de menores repatriados por Estados Unidos según entidad de origen durante 2013. Recuperado de: < http://observatoriocolef.org/Mapa/77>

Oropeza, M. (15 de marzo de 2014). Entrevista a Mónica Oropeza/Entrevistador: J. A. Mena. Copia en posesión del autor.

Pérez, C. (29 de diciembre de 2012). Los "polleritos", otro fenómeno de riesgo para los menores migrantes hacia EU. La Jornada. Recuperado de: < http:// www.jornada.unam.mx/2012/12/29/politica/012n1pol>

Perona, N., Crucella, C., Rocchi, G. y Robin, S. (2000). Vulnerabilidad y exclusión social. Una propuesta metodológica para el estudio de las condiciones de vida de los hogares. Ponencias, Congreso Internacional de Políticas Sociales, Concepción, Chile. Recuperado de: < http://www.ubiobio.cl/cps/ponencia/doc/p15.4.htm>

Petit, J. M. (2003). Migraciones, vulnerabilidad y políticas públicas. Impacto sobre los niños, sus familias y sus derechos. Santiago de Chile: CELADE, BID.

Programa de Defensa e Incidencia Binacional. (2012). Solicitud de opinión consultiva sobre niñez migrante ante la Corte Interamericana de Derechos Humanos, Amicus curiae. Tijuana, B.C: Autor. 
José A. Moreno Mena y Rosa María Avedaño Millán / Arrinconados por la realidad: Menores de circuito

Programa de Defensa e Incidencia Binacional. (2013a). Segundo informe sobre violaciones a derechos humanos de migrantes mexicanos detenidos en los Estados Unidos, 2011-2012. México: Iniciativa Frontera Norte.

Programa de Defensa e Incidencia Binacional. (2013b). Solicitud al DIF por medio del Instituto Federal de Acceso a la Información (IFAI). México: Mimeo, julio.

Pulgarin, M. (2010). Disminuyen casos de menores "de circuito". Infonor Diario Digital, 4 de noviembre. Recuperado de: <http://www.infonor.com.mx/index. $\mathrm{php} /$ norte/10-norte/33246-disminuyen-casos-de-menores->

Ramírez, S., García, J. O., Muñoz, R. G. y Enciso, P. J. (2009). Más allá de la frontera, la niñez migrante: Son las niñas y niños de todos. Estudio exploratorio sobre la protección de la niñez migrante repatriada en la frontera norte. México: Caminos Posibles Investigación, Capacitación y Desarrollo, Sedesol, Indesol.

Ramos, M. E. (2009). Entre la tristeza y la esperanza: Reconstrucciones identitarias de los mexicanos en Estados Unidos. En M. E. Ramos (Coord.), Migración, identidad, emociones, familia (pp. 37-70). México: Fondo Editorial Nuevo León.

Red Consular de la Dirección General de Protección a Mexicanos en el Exterior de la Secretaría de Relaciones Exteriores (2013). Repatriación de menores no acompañados 2012 y 2013. Recuperado de: <https://www.sre.gob.mx/proteccionconsular/images/stories/documentos/Estadisticas_PME/NNA2013.pdf>

Ruiz, O. (2004). Violencia sexual: El caso de las migrantes centroamericanas en la frontera sur. En T. Fernández de Juan (Coord.), Violencia contra la mujer en México (pp. 85-95). México: Comisión Nacional de Derechos Humanos.

Torrado M-P., E. (2012). Las migraciones de menores no acompañados desde una perspectiva de género. Dilemata. Revista Internacional de Éticas Aplicadas, (10), septiembre, 65-84.

Valdéz-Gardea, G. C. (Coord.) (2008). Achicando futuros. Hermosillo: El Colegio de Sonora.

Valdéz-Gardea, G. C. (Coord.) (2013). Memoria digital del IV Encuentro Internacional Migración y Niñez Migrante. Migración y Retorno: Retos para la Familia Migrante. Hermosillo: El Colegio de Sonora.

Vilaboa, E. (2006). Caracterización de la niñez migrante en la frontera norte de México. Los casos de Tijuana y Nogales, México. Suecia: Corredor Bilateral para la Protección de Derechos Humanos, Save the Children. 